\title{
MATRIX VALUED CONJUGATE CONVOLUTION OPERATORS ON MATRIX VALUED $L^{p}$-SPACES
}

\author{
ALI EBADIAN* AND ALI JABBARI
}

Abstract. Let $G$ be a locally compact group equipped with the left Haar measure $m_{G}, M_{n}$ be an $n \times n$ matrix with entries in $\mathbb{C}$ and let $M\left(G, M_{n}\right)$ be the Banach algebra consisting all $M_{n}$-valued measures on $G$. We define the left and right conjugate convolution operators on $L^{p}\left(G, M_{n}\right)$ and characterize these operators. Moreover, we give some necessary and sufficient conditions, in terms of conjugate convolution, for a bounded operator on $L^{p}\left(G, M_{n}\right)$ to be translation invariant. $46 \mathrm{G} 10$.

Mathematics subject classification (2020): Primary 46A32; Secondary 43A05, 43A15, 43A25, 28B05,

Keywords and phrases: Conjugate convolution, locally compact group, matrix valued measure, $p$ convolution operator.

\section{REFERENCES}

[1] C.-H. CHU, Matrix-valued harmonic functions on groups, J. Reine Angew. Math., 552, (2002), 15-52.

[2] C.-H. CHU, Matrix convolution operators on groups, Lecture Notes in Mathematics, Springer-Verlag Berlin Heidelberg, 2008.

[3] C.-H. ChU AND A. T.-M. LAU, Jordan structures in harmonic functions and Fourier algebras on homogeneous spaces, Math. Ann., 336, (2006), 803-840.

[4] A. DerighetTI, Convolution operators on groups, Lecture Notes of the Union Mathematica Italiana, Springer, 2011

[5] J. Diestel and J. J. Uhl, Vector measures, Math. Surv. 15, Amer. Math. Soc., 1977.

[6] A. EBAdian And A. JabBari, Matrix valued p-convolution operators, Oper. Mat., 14, 1 (2020), $117-128$.

[7] L. HÖRMANDER, Estimates for translation invariant operators in $L^{p}$ spaces, Acta Math., 104, 1-2 (1960), 93-140.

[8] A. GHAFFARI, Operators which commute with the conjugation operators, Houston J. Math., 34, 4 (2008), 1225-1232.

[9] A. GhafFari, Conjugate convolution operators and inner amenability, Bull. Belg. Math. Soc. Simon Stivin, 19, 1 (2012), 29-39.

[10] A. JabBari, Positive type and positive definite functions on matrix valued group algebras, Results Math., 75, 4 (2020), Art. N. 149 (25 pages), https://doi.org/10.1007/s00025-020-01278-1.

[11] B. MohammadZadeh, Operators which commute with the conjugation convolution operators, Proc. Roman. Acad. Series A, 17, 1 (2016), 11-15.

[12] C. K. YuAN, Conjugate convolutions and inner invariant means, J. Math. Anal. Appl., 157, 1 (1991), $166-178$. 